\title{
openheart Incidence and predictors of prosthesis- patient mismatch after TAVI using SAPIEN 3 in Asian: differences between the newer and older balloon- expandable valve
}

\author{
Masaki Miyasaka (D , ,' Norio Tada, ${ }^{1}$ Masataka Taguri, ${ }^{1,2}$ Shigeaki Kato, ${ }^{1,3}$ \\ Yusuke Enta (D) , ${ }^{1}$ Masaki Hata, ${ }^{1}$ Yusuke Watanabe (D) , ${ }^{4}$ Toru Naganuma, ${ }^{5}$ \\ Masahiro Yamawaki, ${ }^{6}$ Futoshi Yamanaka, ${ }^{7}$ Shinichi Shirai, ${ }^{8}$ Hiroshi Ueno, ${ }^{9}$ \\ Kazuki Mizutani, ${ }^{10}$ Minoru Tabata, ${ }^{11}$ Kensuke Takagi, ${ }^{12}$ Masanori Yamamoto (D) , ${ }^{13}$ \\ Kentaro Hayashida, ${ }^{14}$ on behalf of the OCEAN-TAVI investigators
}

\begin{abstract}
- Additional material is published online only. To view please visit the journal online (http://dx.doi.org/10.1136/ openhrt-2020-001531).
\end{abstract}

To cite: Miyasaka M, Tada N, Taguri M, et al. Incidence and predictors of prosthesis-patient mismatch after TAVI using SAPIEN 3 in Asian: differences between the newer and older balloon-expandable valve. Open Heart 2021;8:e001531.

doi:10.1136/

openhrt-2020-001531

Received 28 November 2020 Revised 1 February 2021 Accepted 5 March 2021
Check for updates

(c) Author(s) (or their employer(s)) 2021. Re-use permitted under CC BY-NC. No commercial re-use. See rights and permissions. Published by BMJ.

For numbered affiliations see end of article.

Correspondence to Dr Masaki Miyasaka; masaki108@gmail.com

\section{ABSTRACT}

Background The balloon-expandable SAPIEN 3 (S3) is superior to the older-generation balloon-expandable SAPIEN XT (XT) in a lower incidence of paravalvular aortic regurgitation, lower complication rates and better survival in transcatheter aortic valve implantation (TAVI). However, prosthesis-patient mismatch (PPM) more frequently occurs in S3 than XT. Further, little information is available on PPM after TAVI using S3 in Asians. This study aims to determine the incidence and predictors of PPM in S3 by focusing on the difference between S3 and XT using data from a Japanese multicentre registry.

Methods From the Optimised transCathEter vAlvular iNtervention-TAVI (OCEAN-TAVI) registry, 2134 patients undergoing TAVI using S3 or XT were included. PPM was defined as moderate if $\geqq 0.65$ but $\leqq 0.85 \mathrm{~cm}^{2} / \mathrm{m}^{2}$ or severe if $<0.65 \mathrm{~cm}^{2} / \mathrm{m}^{2}$ at the indexed effective orifice area by postprocedural echocardiography.

Results The incidence of moderate and severe PPM in S3 was $13.3 \%$ and $1.3 \%$, respectively. The $20 \mathrm{~mm}$ transcatheter heart valve (THV) was more frequently used in S3 than XT $(7.4 \%$ vs $2.4 \%, p<0.0001)$. PPM was more frequently observed in S3 than XT $(14.7 \%$ vs $8.8 \%, p<0.0001)$. Multivariate logistic regression analysis revealed S3 predicted PPM (OR $1.92(95 \% \mathrm{Cl} 1.35$ to 2.74), $p=0.0003$ ). The mutual predictors for PPM between S3 and XT were younger age, larger body surface area, smaller aortic valve area, no balloon postdilatation and the use of $20 \mathrm{~mm}$ and $23 \mathrm{~mm}$ THV. When comparing $23 \mathrm{~mm}$, $26 \mathrm{~mm}$ and $29 \mathrm{~mm} \mathrm{~S} 3$, the ORs of $20 \mathrm{~mm} \mathrm{S3}$ were 5.67 (95\% Cl 2.88 to 11.12 ), 19.24 (95\% Cl 8.13 to 46.86 ) and 51.03 (95\% Cl 12.28 to 280.77 ), respectively.

Conclusions The incidence of PPM after TAVI using S3 was $14.6 \%$ overall in this Asian population. PPM was more frequently observed in S3 than XT. A considerable number of patients were treated by the $20 \mathrm{~mm} \mathrm{S3}$ in an Asian cohort. The $20 \mathrm{~mm}$ THV was identified as a strong predictor for PPM.

\section{Key questions}

What is already known about this subject?

- Although the balloon-expandable SAPIEN 3 (S3) is superior to the older-generation balloon-expandable SAPIEN XT (XT) in lower complication rates and better survival in transcatheter aortic valve implantation (TAVI), prosthesis-patient mismatch (PPM) more frequently occurs in S3 than XT. However, little information is available on the incidence and predictors of PPM after TAVI using S3 in Asians.

What does this study add?

- According to data from the Japanese multicentre registry, the incidence of moderate and severe PPM after TAVI using S3 was $13.3 \%$ and $1.3 \%$, respectively, which is significantly lower incidences of PPM than those in previous studies from western countries. This study validated that PPM was more frequently observed in S3 than XT in Asians as expected $(14.7 \%$ vs $8.8 \%, p<0.0001)$.

- A smaller-sized transcatheter heart valve (THV) was used in S3 than XT because of the less oversizing design of S3. Therefore, a considerable number of patients $(7.4 \%)$ were treated by the $20 \mathrm{~mm} \mathrm{~S} 3$. Further, the $20 \mathrm{~mm}$ use of THV was identified as a strong predictor for PPM after TAVI using S3. The effect of postdilatation was significant with about $70 \%$ reduction in the occurrence of PPM after TAVI using S3 according to the multivariate logistic regression analysis.

\section{INTRODUCTION}

Prosthesis-patient mismatch (PPM) occurs when the effective orifice area (EOA) of a normally functioning implanted valve prosthesis is small in relation to the patient's body size. A recent study on approximately 60000 patients demonstrated that not only severe 


\section{Key questions}

How might this impact on clinical practice?

- Clinicians should know that a considerable number of $20 \mathrm{~mm} \mathrm{S3}$ is used in transcatheter aortic valve implantation (TAVI) for Asian patients and the use of $20 \mathrm{~mm}$ S3 is a strong risk factor of prosthesis-patient mismatch (PPM). Considering the lack of long-term clinical effect of PPM, transcatheter heart valve selection should be carefully performed in a patient with a small aortic annulus and large body surface area in an era where TAVI is being increasingly performed in younger patients. Postdilatation may be a modifiable variable to reduce the incidence of PPM after TAVI using S3.

but also moderate PPM after surgical aortic valve replacement was associated with higher long-term mortality. ${ }^{1}$ In transcatheter aortic valve implantation (TAVI), a study from the Society of Thoracic Surgeons (STS)/American College of Cardiology transcatheter valve therapy (TVT) registry showed that severe PPM was associated with increased 1-year mortality, but moderate PPM was not. ${ }^{2}$ The long-term clinical impact of moderate PPM after TAVI remains unclear. Therefore, PPM after TAVI remains an issue.

The balloon-expandable Edwards SAPIEN 3 (S3) valve and the self-expanding Medtronic Evolut $\mathrm{R}$ have been widely used in the newer generation transcatheter heart valve (THV). ${ }^{3}$ Although a previous study showed that S3 had better survival than Evolut R, ${ }^{4}$ PPM was more frequently observed in S3 with intra-annular design than in Evolut $\mathrm{R}$ with supra-annular design. ${ }^{5}{ }^{6}$ When comparing S3 with the balloon-expandable Edwards SAPIEN XT (XT) valve which is the predecessor model of S3, S3 showed better clinical and survival outcomes and lower incidence rate of complications such as paravalvular aortic regurgitation and vascular complications. ${ }^{7-9}$ However, contrary to expectation, S3 more frequently caused PPM than XT. ${ }^{10-12}$ Further, differences in the predictors for PPM between XT and S3 have not been studied. Thus, some caution is needed in interpreting the results of previous studies regarding PPM after TAVI using balloon-expandable devices. Furthermore, although race difference in PPM may exist, ${ }^{13}{ }^{14}$ limited information is available on PPM after TAVI with S3 in Asians. The aim of this study is to determine the incidence and predictors of PPM by focusing on the differences between S3 and SAPIEN XT (XT) using data from the Japanese multicentre registry.

\section{METHODS}

\section{Study population}

The Optimised transCathEter vAlvular iNterventionTAVI (OCEAN-TAVI) registry is a multicentre prospective registry affiliated with the 14 centres in Japan (online supplemental material). This trial is registered with the University Hospital Medical Information Network (UMIN; UMIN000020423). Between October 2013 and May 2017, a total of 2588 Japanese patients having severe aortic stenosis underwent TAVI using devices including the Edwards SAPIEN XT, Edwards SAPIEN 3 (Edwards Lifesciences, Irvine, California, USA), Medtronic CoreValve, or Medtronic CoreValve Evolut R prosthesis (Medtronic, Minneapolis, MN) were prospectively included in the OCEAN-TAVI registry. ${ }^{15}$ These patients were deemed to be at high risk for surgery by a local heart team. No patient underwent valve-in-valve TAVR. In this study, we excluded 454 patients for the following reasons: use of self-expandable THV including CoreValve $(n=195)$ and CoreValve Evolut R ( $\mathrm{n}=148)$, death before discharge $(n=58)$, conversion to open chest surgery $(n=14)$, use of second THV $(n=14)$, absence of THV as a result of delivery failure $(n=1)$ and unreliable echocardiographic data as a result of left ventricle obstruction or poor image $(n=24)$. The remaining 2134 patients were included in this study.

\section{Procedures}

Detailed TAVI procedures have been previously described. ${ }^{14}$ The prosthesis size was determined on the basis of the findings from multidetector CT and/or echocardiography during a TAVI procedure. The devices were delivered via the transfemoral, trans-subclavian, transaortic or transapical approaches.

\section{Definitions of PPM and area oversizing}

Echocardiographic evaluation was performed at baseline before TAVI and at discharge. PPM was assessed using postprocedural echocardiography. In this study, patients with moderate and severe PPM were included in the PPM group. According to the Valve Academic Research Consortium-2 criteria, ${ }^{16}$ we defined the severity of PPM according to the indexed EOA of the prosthetic valve and classified it as follows: none or mild when $>0.85$ $\mathrm{cm}^{2} / \mathrm{m}^{2}$; moderate when between $0.85 \mathrm{~cm}^{2} / \mathrm{m}^{2}$ and 0.65 $\mathrm{cm}^{2} / \mathrm{m}^{2}$; severe when $<0.65 \mathrm{~cm}^{2} / \mathrm{m}^{2}$. In obese patients (body mass index $\geqq 30 \mathrm{~kg} / \mathrm{m}^{2}$ ), lower criteria were used as follows: none or mild when $>0.70 \mathrm{~cm}^{2} / \mathrm{m}^{2}$; moderate when between $0.70 \mathrm{~cm}^{2} / \mathrm{m}^{2}$ and $0.60 \mathrm{~cm}^{2} / \mathrm{m}^{2}$; and severe when $<0.60 \mathrm{~cm}^{2} / \mathrm{m}^{2}$ because PPM tends to be overestimated in obese patients if the same criteria which is used in lean patients. ${ }^{16} 17$ Area oversizing was determined as follows: (THV nominal area/annulus area -1 ) $\times 100$. The nominal areas of XT and S3 are presented in online supplemental table 1.

\section{Subgroup analysis}

To investigate the relationship between a degree of oversizing and a choice of THV size, a subgroup analysis was performed. Patients with an annulus area between 314 $\mathrm{mm}^{2}$ and $345 \mathrm{~mm}^{2}, 415 \mathrm{~mm}^{2}$ and $430 \mathrm{~mm}^{2}$, or $530 \mathrm{~mm}^{2}$ and $546 \mathrm{~mm}^{2}$ were only included in this analysis (table 1 ).

\section{Statistical analysis}

Quantitative variables were assessed for normal distribution using the Shapiro-Wilk test and are expressed as the mean $\pm \mathrm{SD}$ or as the median and interquartile range (IQR 25\%-75\%), as appropriate. In addition, qualitative 
Table 1 Used prosthesis size in patients with specific ranges of aortic annulus area in which there are discrepancies in recommended size between $\mathrm{S} 3$ and $\mathrm{XT}$

\begin{tabular}{llrll}
\hline & S3 & XT & P value & Annulus area \\
\hline Used prosthesis size & & & & \\
$20 \mathrm{~mm}$ & $18 / 117(15.4 \%)$ & $2 / 213(0.9 \%)$ & $<0.0001$ & $314-345 \mathrm{~mm}^{2}$ \\
$23 \mathrm{~mm}$ & $36 / 65(55.4 \%)$ & $20 / 90(22.2 \%)$ & $<0.0001$ & $415-430 \mathrm{~mm}^{2}$ \\
$26 \mathrm{~mm}$ & $10 / 12(83.3 \%)$ & $7 / 23(30.4 \%)$ & 0.003 & $530-546 \mathrm{~mm}^{2}$ \\
\hline
\end{tabular}

This table shows the result of the subgroup analysis based on the annulus areas in which there are discrepancies in recommended size between S3 and XT. The patients with annulus area between $314 \mathrm{~mm}^{2}$ and $345 \mathrm{~mm}^{2}, 415 \mathrm{~mm}^{2}$ and $430 \mathrm{~mm}^{2}$, or $^{2} 30 \mathrm{~mm}^{2}$ and $546 \mathrm{~mm}^{2}$ were included.

S3, SAPIEN; XT, SAPIEN XT.

variables are expressed as numeric values and percentages. Quantitative variables were compared using the unpaired Student's t-test or Wilcoxon's rank-sum test, depending on the distribution of the variables. The $\chi^{2}$ or Fisher's exact test was used to compare qualitative variables. Univariate and multivariate logistic regression analyses were performed to identify the predictors for moderate and severe PPM. Cumulative survival rates were analysed using the Kaplan-Meier method, and differences were assessed using the log-rank test. To compare the incidence rate of PPM between S3 and XT after adjusting confounding factors which affect the occurrence of PPM, a propensity score matching was applied (figures 1 and 2). ${ }^{18}$ The propensity score was estimated by a logistic regression model using the following factors as the predictors: age, sex (male/female), height, weight, body surface area, body mass index and aortic annulus area measured by CT. Based on the estimated propensity score, we conducted 1:1 matching for patients with and without PPM. All analyses were considered statistically significant at a two-tailed $\mathrm{p}<0.05$. Data were analysed using JMP Pro software, V.12.1.0. (SAS Institute) and R Statistic software, V.3.5.0.

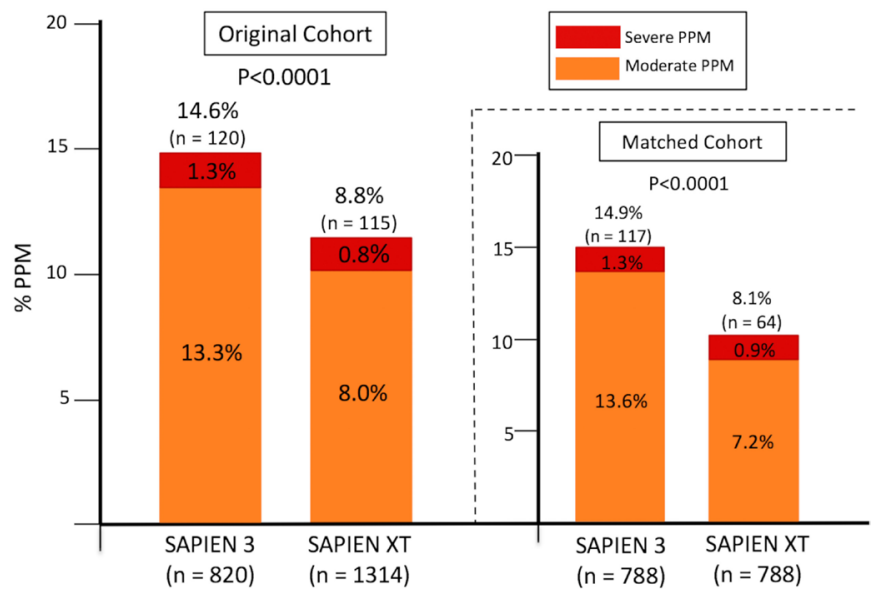

Figure 1 Comparison of S3 and XT in the incidence of PPM in the original and matched cohort. PPM, prosthesis-patient mismatch; S3, SAPIEN 3; XT, SAPIEN XT.

\section{RESULTS}

Of the 2134 patients, 1473 females (69.0\%) were included. The mean age, body surface area (BSA) and STS score were $84.2 \pm 5.2$ years, $1.44 \pm 0.17 \mathrm{~m}^{2}$ and $8.0 \pm 6.4$, respectively. Moderate and severe PPM was observed in $214(10.0 \%)$ and $21(1.0 \%)$ patients, respectively. S3 was approved by Pharmaceuticals and Medical Devices Agency in Japan and replaced XT in 2016. There were $820(38.4 \%)$ and $1314(61.6 \%)$ patients undergoing TAVI using S3 and XT, respectively.

To determine the difference in the incidence of PPM between S3 and XT, propensity score matching was
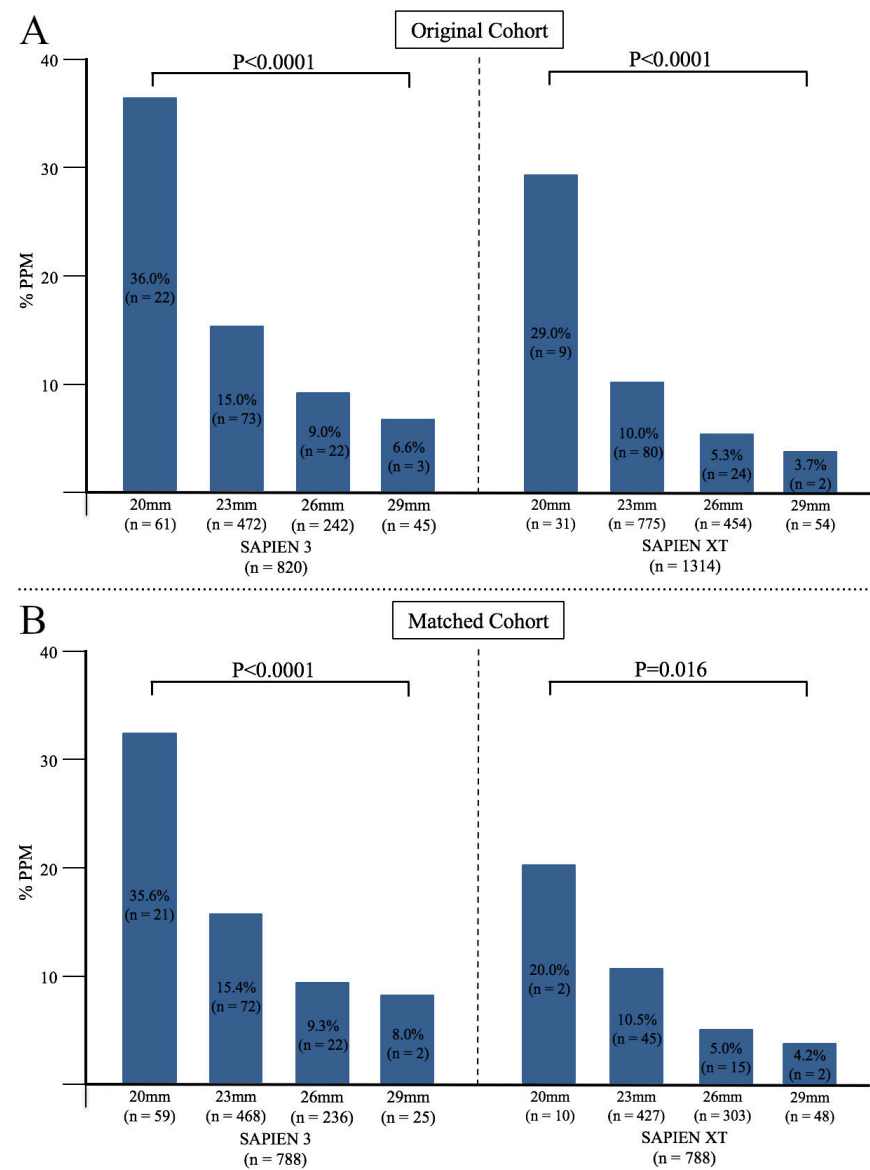

Figure 2 Incidence of PPM in each size of S3 or XT in the original and matched cohort. PPM, prosthesis-patient mismatch; S3, SAPIEN 3; XT, SAPIEN XT. 
applied. Matched groups with similar baseline characteristics were identified (788 patients with S3 and 788 with XT). Patients and procedural characteristics of the original cohort and matched cohort are presented in online supplemental tables 2 and 3, respectively. Patients undergoing TAVI using S3 had lower incidences of major vascular complication and paravalvular aortic regurgitation regardless of the propensity score matching, as expected (online supplemental tables 2 and 3). PPM was more frequently observed in S3 than XT both in the matched cohort and original cohort $(14.9 \%$ vs $8.1 \%, \mathrm{p}<0.0001 \%$ and $14.6 \%$ versus $8.8 \%, \mathrm{p}<0.0001$, respectively) (figure 1), but no statistical difference was observed in the incidence rate of severe PPM between the S3 and the XT cohorts.

Baseline clinical and procedural characteristics of patients with or without PPM in the S3, XT, and entire cohorts are shown in table 2. The PPM group in the S3 cohort had a younger age, greater height, greater weight, larger BSA, lower STS score and smaller aortic valve area than the non-PPM group. In the XT cohort, there were no differences in body-size-related variables except for BSA between the PPM and non-PPM groups. The annuli were smaller in the PPM group than in the non-PPM group, in both the XT and the S3 cohorts (table 2). The smaller-sized THV more frequently caused PPM both in the original and matched cohort (figure 2). Surprisingly, PPM was observed in 21 out of $59(36.0 \%)$ patients who underwent TAVI using $20 \mathrm{~mm}$ S3.

Regarding the association between a degree of oversizing and a choice of THV size, it is summarised in table 1 that the usage of THV's size in patients with specific ranges of aortic annulus area in which there are discrepancies in recommended size of THV between S3 and XT. Smaller-sized THV was more frequently selected in S3 than XT (table 1). Accordingly, the $20 \mathrm{~mm}$ THV was more frequently used in S3 than XT (table 1).

The PPM group in the S3 cohort had a higher frequency of balloon predilatation and a significantly lower frequency of postdilatation (table 2). Postprocedural echocardiography data are presented in table 3. As expected, the PPM groups had a greater mean transprosthetic pressure gradient (PG) and a higher prevalence of transprosthetic $\mathrm{PG} \geqq 20 \mathrm{~mm} \mathrm{Hg}$ than non-PPM groups (table 3). Further, transprosthetic PG $\geqq 20 \mathrm{~mm} \mathrm{Hg}$ was observed only in patients with TAVI with $20 \mathrm{~mm}$ or 23 mm THV (figure 3).

\section{The predictors for PPM}

The multivariate logistic regression analysis for PPM is presented in table 4 . The use of S3 instead of XT was identified as an independent predictor for PPM in the entire cohort (OR 1.92 (95\% CI 1.35 to 2.74 ), $\mathrm{p}=0.0003$ ). The mutual predictors for PPM between the S3 and XT cohorts were younger age, larger BSA, smaller aortic valve area, no balloon postdilatation, and the use of 20 $\mathrm{mm}$ and $23 \mathrm{~mm}$ THV (table 4). Body mass index was not associated with PPM in either group. The ORs for PPM with postdilatation vs without postdilatation in the $\mathrm{S} 3$ and the XT cohorts were 0.30 (95\% CI 0.14 to $0.60, \mathrm{p}=0.0003$ ) and 0.51 (95\% CI 0.28 to $0.89, \mathrm{p}=0.02$ ), respectively. The effect of postdilatation was significant with about $70 \%$ reduction in the occurrence of PPM in the S3 cohort (table 4). There was no interaction between 'aortic valve area and predilatation', 'aortic valve area and postdilatation' or 'predilatation and postdilatation'. To assess potential multicollinearity among variables used in the multivariate logistic regression analyses, the variance inflation factor was calculated. The largest value of the variance inflation factor was 2.32 which was observed in BSA. Values of the variance inflation factor for the other variables used were less than 1.90. These findings confirmed that there was no significant multicollinearity in this analysis.

\section{Cumulative mortality and PPM}

At a median follow-up duration of 721 days (IQR 396-928 days), a total of 365 patients died in the entire cohort. The Kaplan-Meier estimates of cumulative all-cause and cardiovascular mortality in the two groups based on the presence of any-PPM are shown in online supplemental figure 1. The Kaplan-Meier estimates in the three groups based on PPM severity are shown in online supplemental figure 2. There was no difference in the probability of cumulative mortality over the entire follow-up period after TAVI between the two groups in each cohort, except for all-cause mortality in the S3 cohort (online supplemental figure 1C). In the S3 cohort, the PPM group had rather lower cumulative mortality than the non-PPM group. However, multivariate cox regression analysis revealed that PPM has no effect on the mortality in the $\mathrm{S} 3$ cohort (HR $0.58,95 \%$ CI 0.22 to $1.28, \mathrm{p}=0.19$ ), where the following variables were included: PPM, sex, age, body mass index, clinical frailty score, the concentration of serum haemoglobin, estimated glomerular filtration rate, left ventricular ejection fraction, STS score, preprocedural mitral regurgitation $\geq$ moderate and paravalvular aortic regurgitation $\geq$ moderate.

\section{DISCUSSION}

In this study, there were four clinical observations. First, the incidence of PPM after TAVI using S3 was 14.6\% overall in this Asian population. Second, PPM in S3 was more frequently observed than that in XT $(14.6 \%$ vs $8.8 \%, \mathrm{p}<0.0001)$. Third, the smaller-sized THV tended to be selected in S3 than XT. Last, the use of $20 \mathrm{~mm}$ THV was identified as a strong predictor for PPM in S3.

Data on Asian in TAVI remain insufficient. According to a study from the TVT registry, among 70221 patients who underwent TAVI, the Asian race accounted for less than $1.5 \%$ of the entire cohort. ${ }^{19}$ Further, although the differences in PPM across races may exist, ${ }^{13} 14$ limited information on PPM after TAVI in Asian is available. Regarding PPM after TAVI in Asian patients, although our previous work included the largest numbers of Asian patients 


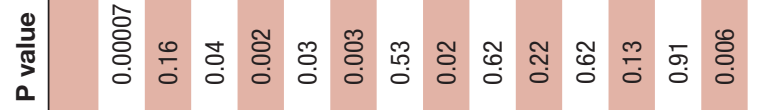

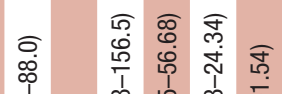

द्ष

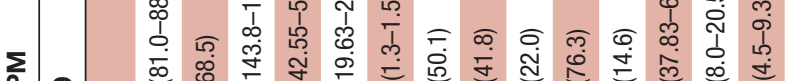

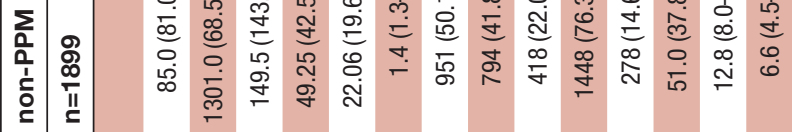
(1)

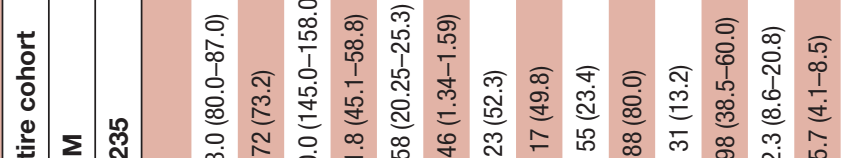

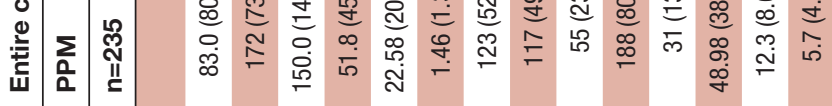

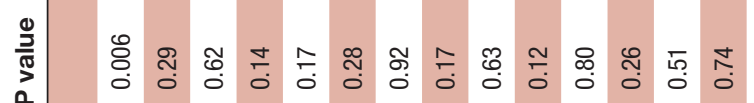

$\frac{0}{0}$

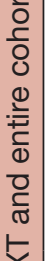

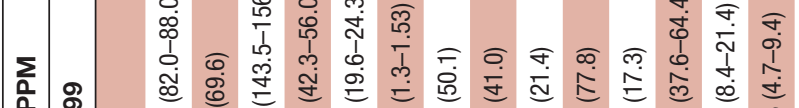

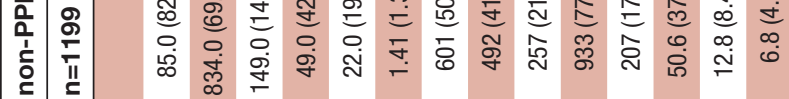

๗்

$\stackrel{\oplus}{\rightleftarrows}$

$\sum_{\substack{n \\ \hline}} \frac{t}{0}$

$\sum_{0}$

蒿

흔

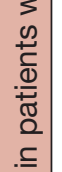

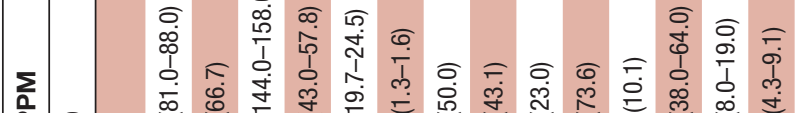

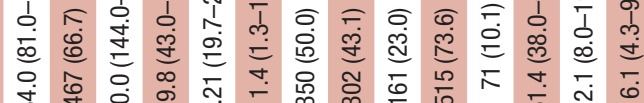

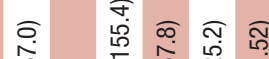

के $\overline{0}$ क

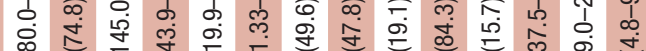

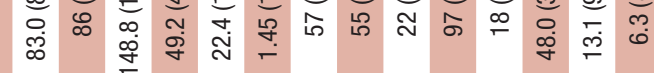

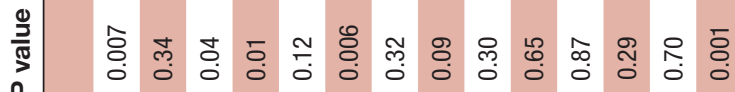

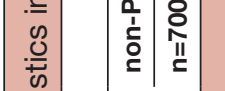

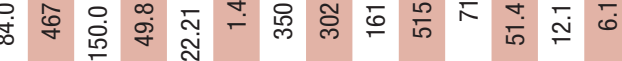

o. लु.

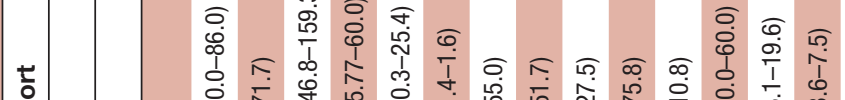

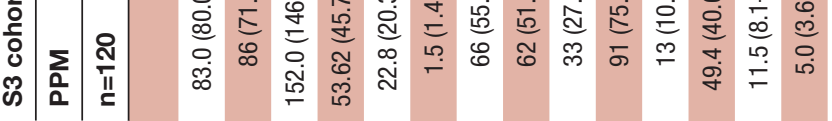

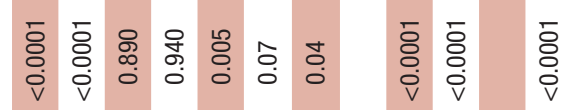

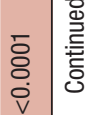

站

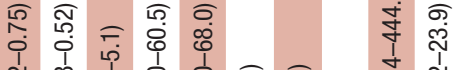

范

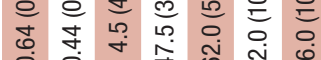

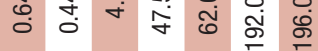

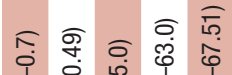

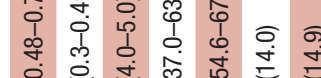

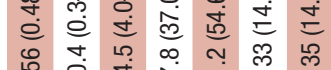

菅

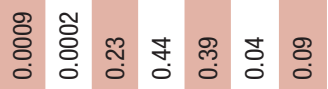

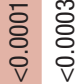

চ⿳亠口冋.

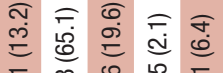

ฮู่

id

สิ ฮิ สิ

एँ.

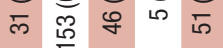

న.

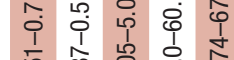

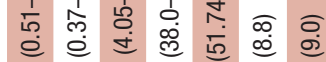

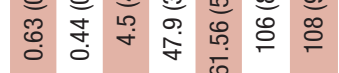

동

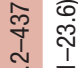

花

का

స్ల

क $\bar{\sigma}$ ल

每

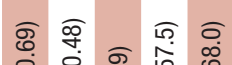

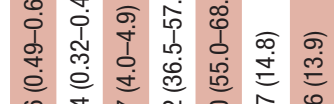

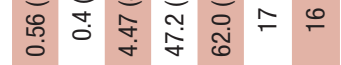

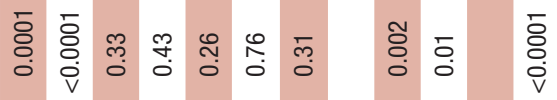

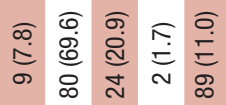

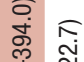

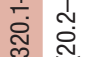

깅

ळั

হ.

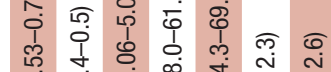

过

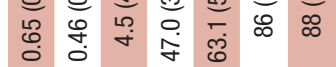

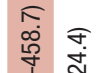

d.

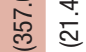

กั กิ

बิ

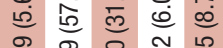

总

हิธ สิ

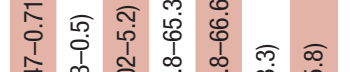

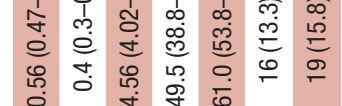

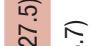

I

लृ

당

ल $\bar{\infty} \widehat{\infty} \widehat{\infty}$

$\stackrel{\infty}{=}$ 远

ส 


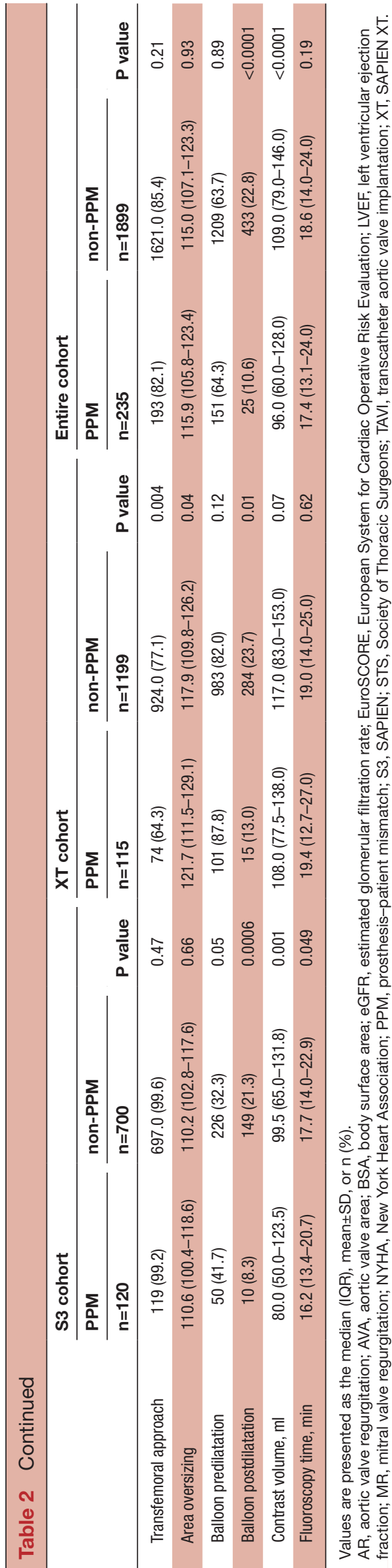

among the available literature, the study included only 139 patients who underwent TAVI using S3. ${ }^{14}$ Thus, the incidence of PPM after TAVI using S3 remained unclear. In contrast, the current study included a considerable number of patients and first determined the incidence of PPM after TAVI using S3 in Asian patients. The incidence of PPM in S3 was $14.6 \%$ in this Asian population, which was significantly lower than reported incidences of $45.0 \%$ to $47.8 \%$ in previous studies, ${ }^{1120} 21$ possibly because Asians have a greater annulus to BSA ratio than Caucasians. ${ }^{14}$

It has been reported that S3 more frequently caused PPM in the previous studies from western countries with small numbers of patients. ${ }^{10}{ }^{20}$ Similarly, the current study ascertained that S3 caused PPM about 1.92 times more than XT even in the Asian cohort (table 3 and figure 1). The structural differences between S3 and XT were the polyethylene terephthalate outer sealing skirt and the shape of the stent frame. Although the exact cause of the higher incidence of PPM in S3 than XT remains unclear, three possible causes were occupying annular space by additional material of the outer skirt, lower area oversizing design of S3, and the hyperboloid shape of S3. The outer sealing skirt of S3 significantly reduces paravalvular aortic regurgitation and allows a lesser degree of oversizing of S3 than XT. ${ }^{7-10}$ However, the presence of supplemental material of the outer skirt potentially occupying the annular space may cause PPM. ${ }^{10}$ Second, compared with XT, S3 needs a lesser degree of CT area oversizing according to the manufacturer (online supplemental table 1) ${ }^{22}$ In other words, a smaller-sized THV was more frequently used in S3 than XT. For example, in a TAVI procedure in a patient with an annulus area of $320 \mathrm{~mm}^{2}$, a $20 \mathrm{~mm}$ THV would be selected when S3 was used, but a $23 \mathrm{~mm}$ THV would be selected when XT (table 1). The feature of "the less oversizing design of S3' was known as a reason for a low incidence of annulus rupture in S3. At the same time, the feature may be a cause of PPM in S3. Indeed, PPM was more frequently observed in smaller sized THV (figure 2). Last, regarding the hyperboloid shape of S3, some studies using postprocedural CT demonstrated that implanted S3 had a flared THV inflow shape, and the narrowest THV area was observed in the midportion of THV. Lower EOA in S3 might be attributed to a smaller THV area of the midportion of THV. ${ }^{23}{ }^{24}$ Furthermore, it should be noted that the feature of the outer sealing skirt is strengthened and inherited by the newest balloon-expandable valve, SAPIEN 3 ULTRA valve (Edwards Lifesciences), ${ }^{25}$ which remains unavailable in Japan. The device sizing chart of SAPIEN 3 ULTRA is identical to that of S3, and the less oversizing design is also inherited. These features of SAPIEN 3 ULTRA would theoretically be a cause of PPM. Further studies to address this issue is warranted.

Regarding the analysis of the predictors for PPM, this study showed that some mutual predictors in the S3 and XT cohorts were identified: younger age, larger BSA, smaller aortic valve area and smaller-sized THV. Although these factors have been already reported in previous 


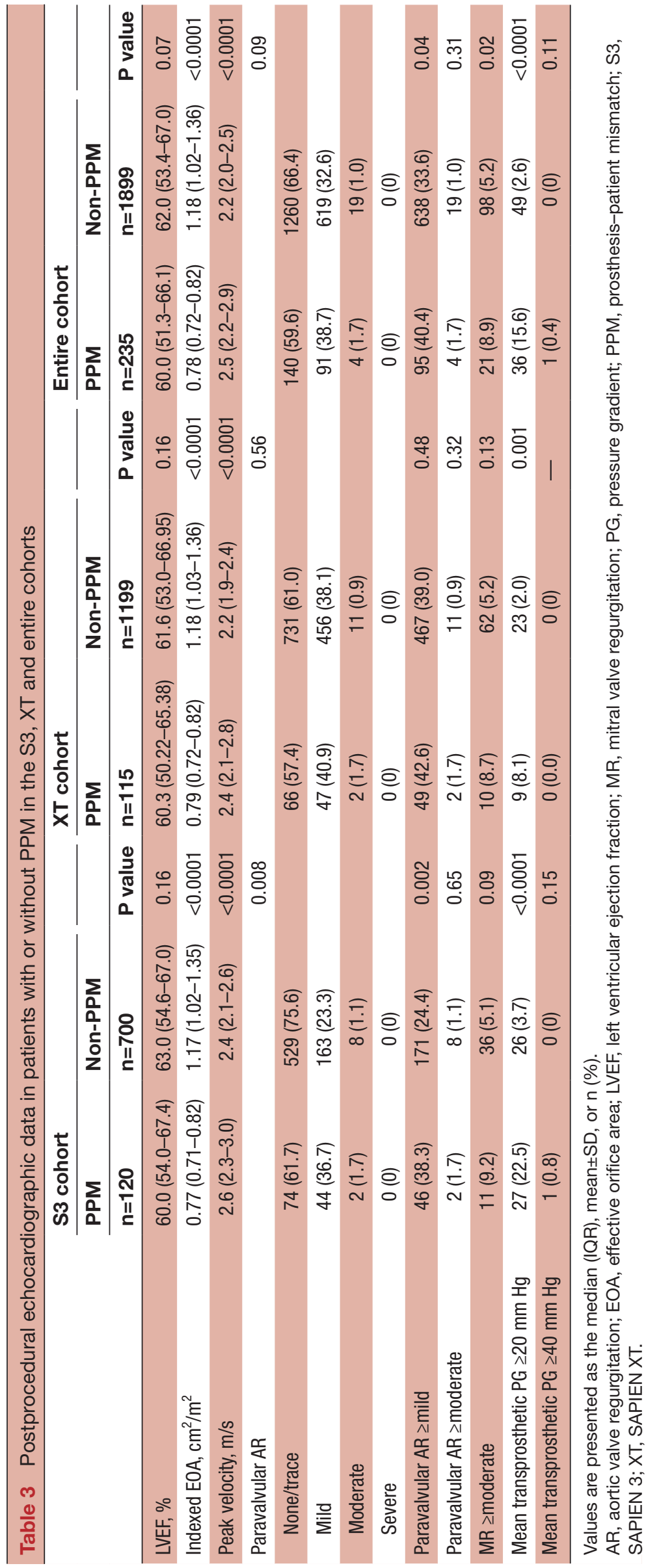




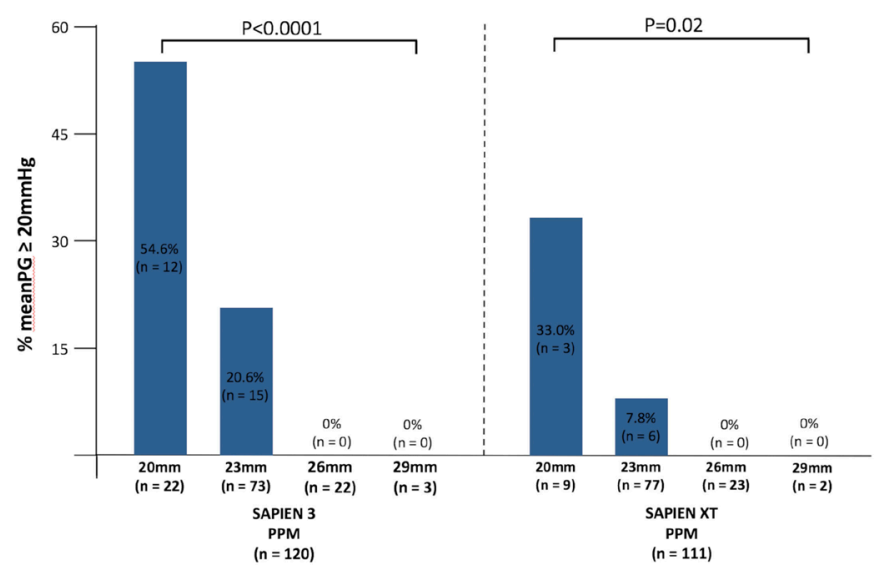

Figure 3 Prevalence of transprosthetic mean PG $\geq 20 \mathrm{~mm}$ $\mathrm{Hg}$ by postprocedural echocardiography in each size of $\mathrm{S} 3$ or XT among patients with PPM. PG, pressure gradient; PPM, prosthesis-patient mismatch; S3, SAPIEN 3; XT, SAPIEN XT.

studies, ${ }^{2} 1214$ it was highlighted in this study that the use of $20 \mathrm{~mm}$ THV and $23 \mathrm{~mm}$ THV were identified as predictors for PPM. Particularly, the use of $20 \mathrm{~mm}$ THV was identified as the powerful predictor as the OR of 20 $\mathrm{mm}$ THV when compared with $23 \mathrm{~mm}, 26 \mathrm{~mm}$, and 29 mm THV was 5.67, 19.24 and 51.03, respectively. It may be likely that this finding was not a significant problem because the percentages of use of $20 \mathrm{~mm} \mathrm{S3}$ was low of $1.9 \%$ to $3.2 \%$ in previous studies from Western countries. ${ }^{8} 1321$ However, the use of $20 \mathrm{~mm} \mathrm{S3}$ was indeed observed in $7.8 \%$ in this Asian cohort, possibly because of Asians' smaller aortic annulus. ${ }^{14627}$ Furthermore, about one-third of patients with $20 \mathrm{~mm} \mathrm{S3}$ developed PPM in this study (figure 2). Considering these findings, clinicians should know that $20 \mathrm{~mm} \mathrm{~S} 3$ is used more frequently in Asians than Caucasians, and PPM likely to occur even in Asian patients. Another finding in the multivariate analysis in this study is that the effect of postdilatation was significant with about $70 \%$ reduction in the occurrence of PPM in the S3 cohort (table 4). A previous study by Hahn $e t a l$ showed the association between conducting postdilatation and larger EOA by postprocedural echocardiography but failed to demonstrate statistical significance on the association between post-dilation and the occurrence of PPM after TAVI using S3. ${ }^{21}$ Postdilatation may be more important in TAVI with S3 than XT in terms of PPM.

Not only any PPM but also severe did not predict allcause mortality in the S3, XT or entire cohort in this study. This study may be statistically underpowered to determine the effect of severe PPM on mortality, according to the previous study. ${ }^{2}$ This study included only 22 patients with severe PPM. Not only was the entire sample size in this study smaller than the study from the TVT registry, ${ }^{2}$ but also the incidence rate of severe PPM was 10 times lower than that in their study. Thus, the impact of severe PPM of S3 on mortality was inconclusive in this study. Regarding other clinical outcomes, it should be noted that the prevalence of the mean transprosthetic $P G \geq 20$ $\mathrm{mm} \mathrm{Hg}$ by postprocedural echocardiography was significantly higher in the S3 than in the XT cohort (table 3). Notably, $54.6 \%$ of patients with PPM after TAVI using 20 $\mathrm{mm}$ S3 had the mean transprosthetic PG $\geq 20 \mathrm{~mm} \mathrm{Hg}$ (figure 3). This finding would be a potential issue associated with poor long-term clinical outcomes, particularly in a lower surgical risk population. ${ }^{28}$ Considering the lack of long-term clinical effect of PPM, THV selection should be carefully performed when TAVI using S3 is performed in a patient with a small aortic annulus and large BSA in an era where TAVI is being increasingly performed in

Table 4 Multivariate logistic regression analysis for the predictors of PPM in the S3, XT and entire cohorts

\begin{tabular}{|c|c|c|c|c|c|c|}
\hline & \multicolumn{2}{|l|}{ S3 cohort } & \multicolumn{2}{|l|}{ XT cohort } & \multicolumn{2}{|l|}{ Entire cohort } \\
\hline & OR (95\% CI) & $P$ value & OR (95\% CI) & $P$ value & OR (95\% Cl) & $P$ value \\
\hline Age, years & 0.94 (0.9 to 0.98$)$ & 0.003 & 0.96 (0.92 to 0.99$)$ & 0.02 & 0.95 (0.92 to 0.98$)$ & 0.0003 \\
\hline Body mass index, $\mathrm{kg} / \mathrm{m}^{2}$ & $0.93(0.86$ to 1.00$)$ & 0.06 & 0.99 (0.92 to 1.05$)$ & 0.68 & $0.96(0.91$ to 1.01$)$ & 0.10 \\
\hline AVA, $\mathrm{cm}^{2}$ (per $0.1 \mathrm{~cm}^{2}$ increase) & $0.74(0.64$ to 0.85$)$ & $<0.0001$ & 0.81 (0.70 to 0.92$)$ & 0.002 & 0.77 (0.70 to 0.85$)$ & $<0.0001$ \\
\hline Balloon predilatation & 1.17 (0.75 to 1.83$)$ & 0.49 & 1.39 (0.79 to 2.62) & 0.27 & 1.27 (0.9 to 1.81$)$ & 0.17 \\
\hline Balloon postdilatation & $0.30(0.14$ to 0.60$)$ & 0.0003 & 0.51 (0.28 to 0.89$)$ & 0.02 & 0.41 (0.25 to 0.62$)$ & $<0.0001$ \\
\hline 20 mm valve (vs 23 mm valve) & 5.67 (2.88 to 11.12$)$ & $<0.0001$ & 6.27 (2.5 to 14.81$)$ & 0.0002 & 5.26 (3.09 to 8.83) & $<0.0001$ \\
\hline $20 \mathrm{~mm}$ valve (vs $26 \mathrm{~mm}$ valve) & 19.24 (8.13 to 46.86$)$ & $<0.0001$ & 21.83 (7.52 to 62.10$)$ & $<0.0001$ & 18.49 (9.6 to 35.85$)$ & $<0.0001$ \\
\hline $20 \mathrm{~mm}$ valve (vs $29 \mathrm{~mm}$ valve) & 51.03 (12.28 to 280.77$)$ & $<0.0001$ & 47.36 (9.18 to 371.34$)$ & $<0.0001$ & 43.07 (14.66 to 149.18$)$ & $<0.0001$ \\
\hline $26 \mathrm{~mm}$ valve (vs $29 \mathrm{~mm}$ valve) & $2.65(0.79$ to 12.41$)$ & 0.12 & $2.17(0.59$ to 14.03$)$ & 0.27 & 2.33 (0.94 to 7.08$)$ & 0.07 \\
\hline SAPIEN3 (vs SAPIENXT) & - & - & - & - & 1.92 (1.35 to 2.74$)$ & 0.0003 \\
\hline
\end{tabular}

AVA, aortic valve area; BSA, body surface area; PPM, prosthesis-patient mismatch; S3, SAPIEN 3; XT, SAPIEN XT. 
younger patients. Thus, an alternative device for TAVI to overcome the issue regarding PPM is warranted. In the meanwhile, postdilatation might be a modifiable variable to reduce the incidence of PPM after TAVI using S3.

Some limitations should be acknowledged. Errors can occur when estimating the prosthetic valve EOA by Doppler echocardiography as a result of the difficulty in measuring the stroke volume in the left ventricular outflow tract. ${ }^{12}$ There was no echocardiography core lab for this study. Selection bias might exist regarding postdilatation because whether conducting postdilatation was at operator's discretion. Furthermore, we did not investigate the advantages and disadvantages of postdilatation.

\section{CONCLUSIONS}

The incidence of PPM after TAVI with S3 in this Asian cohort was lower than that in previous studies including Caucasians (Moderate PPM: 13.3\%, severe PPM: 1.3\%). The incidence of PPM after TAVI using S3 was significantly higher than that using XT. The smaller-sized THV tended to be selected in S3 than XT because of the less oversizing design of S3, and PPM was more frequently observed in smaller sized THV. The mutual predictors for PPM between S3 and XT were younger age, larger BSA, smaller aortic valve area, no balloon postdilatation and the use of smaller-sized THV. Particularly, the use of $20 \mathrm{~mm}$ THV was identified as a strong predictor for PPM after TAVI using S3.

\section{Author affiliations}

${ }^{1}$ Cardiovascular Center, Sendai Kosei Hospital, Sendai, Miyagi, Japan

${ }^{2}$ Department of Biostatistics, Yokohama City University School of Medicine,

Yokohama, Kanagawa, Japan

${ }^{3}$ Center for Regional Cooperation Iwaki, Meisei University, Iwaki, Fukushima, Japan

${ }^{4}$ Department of Internal Medicine, Teikyo University Hospital, Itabashi-ku, Tokyo,

Japan

${ }^{5}$ Interventional Cardiology Unit, New Tokyo Hospital, Matsudo, Chiba, Japan

${ }^{6}$ Department of Cardiovascular Medicine, Saiseikai Yokohamashi Tobu Hospital,

Yokohama, Japan

${ }^{7}$ Department of Cardiovascular Medicine, Shonankamakura General Hospital,

Kamakura, Kanagawa, Japan

${ }^{8}$ Cardiology, Kokura Mem Hosp, Kitakyushu, Fukuoka, Japan

${ }^{9}$ Cardiovascular Medicine, University of Toyama University Hospital, Toyama,

Toyama, Japan

${ }^{10}$ Department of Cardiovascular Medicine, Osaka City University Graduate School of Medicine, Osaka, Japan

${ }^{11}$ Department of Cardiovascular Surgery, Tokyo Bay Urayasu Ichikawa Medical Center, Chiba, Japan

${ }^{12}$ Department of Cardiology, Ogaki Municipal Hospital, Ogaki, Gifu, Japan

${ }^{13}$ Interventional Cardiology, Toyohashi Heart Center, Toyohashi, Aichi, Japan

${ }^{14}$ Department of Cardiology, Keio University School of Medicine, Tokyo, Japan

Acknowledgements The authors thank the investigators and institutions that have participated in the OCEAN-TAVI registry.

Contributors All authors have made substantial contributions to the study, and all authors endorse the data and conclusions.

Funding The OCEAN-TAVI registry is supported by Edwards Lifesciences, Medtronic, and Daiichi-Sankyo company.

Competing interests NT, MY, TN, SS, KM, MT, HU and YW are clinical proctors for Edwards Lifesciences and Medtronic. KT and KH are clinical proctors of Edwards Lifesciences.
Patient consent for publication Not required.

Ethics approval The OCEAN-TAVI registry was registered with the University Hospital Medical Information Network Clinical Trial Registry and accepted by the International Committee of Medical Journal Editors (UMIN-ID: 000020423).

Provenance and peer review Not commissioned; externally peer reviewed.

Data availability statement No data are available. The data in this research are deidentified participant data. The data, materials will not be available to researchers for purpose of reproducing the results or replicating the procedure.

Open access This is an open access article distributed in accordance with the Creative Commons Attribution Non Commercial (CC BY-NC 4.0) license, which permits others to distribute, remix, adapt, build upon this work non-commercially, and license their derivative works on different terms, provided the original work is properly cited, appropriate credit is given, any changes made indicated, and the use is non-commercial. See: http://creativecommons.org/licenses/by-nc/4.0/.

\section{ORCID iDs}

Masaki Miyasaka http://orcid.org/0000-0001-5490-7925

Yusuke Enta http://orcid.org/0000-0003-1931-1012

Yusuke Watanabe http://orcid.org/0000-0003-2297-5303

Masanori Yamamoto http://orcid.org/0000-0001-5210-6382

\section{REFERENCES}

1 Fallon JM, DeSimone JP, Brennan JM, et al. The incidence and consequence of prosthesis-patient mismatch after surgical aortic valve replacement. Ann Thorac Surg 2018;106:14-22.

2 Herrmann HC, Daneshvar SA, Fonarow GC, et al. ProsthesisPatient Mismatch in Patients Undergoing Transcatheter Aortic Valve Replacement: From the STS/ACC TVT Registry. J Am Coll Cardiol 2018;72:2701-11.

3 Grover FL, Vemulapalli S, Carroll JD, et al. 2016 Annual Report of The Society of Thoracic Surgeons/American College of Cardiology Transcatheter Valve Therapy Registry. J Am Coll Cardiol 2017;69:1215-30.

4 Deharo P, Bisson A, Herbert J, et al. Impact of Sapien 3 BalloonExpandable versus Evolut R Self-Expandable transcatheter aortic valve implantation in patients with aortic stenosis: data from a nationwide analysis. Circulation 2020;141:260-8.

5 Okuno T, Khan F, Asami M, et al. Prosthesis-patient mismatch following transcatheter aortic valve replacement with supraannular and intra-annular prostheses. JACC Cardiovasc Interv 2019;12:2173-82.

6 Lanz J, Kim W-K, Walther T, et al. Safety and efficacy of a self-expanding versus a balloon-expandable bioprosthesis for transcatheter aortic valve replacement in patients with symptomatic severe aortic stenosis: a randomised non-inferiority trial. Lancet 2019;394:1619-28.

7 Leon MB, Smith CR, Mack MJ, et al. Transcatheter or surgical aortic-valve replacement in intermediate-risk patients. $N$ Engl $J$ Med 2016;374:1609-20.

8 Herrmann HC, Thourani VH, Kodali SK, et al. One-year clinical outcomes with SAPIEN 3 transcatheter aortic valve replacement in high-risk and inoperable patients with severe aortic stenosis. Circulation 2016;134:130-40.

9 Kodali S, Thourani VH, White J, et al. Early clinical and echocardiographic outcomes after SAPIEN 3 transcatheter aortic valve replacement in inoperable, high-risk and intermediate-risk patients with aortic stenosis. Eur Heart J 2016;37:2252-62.

10 Theron A, Pinto J, Grisoli D, et al. Patient-prosthesis mismatch in new generation trans-catheter heart valves: a propensity score analysis. Eur Heart J Cardiovasc Imaging 2018;19:225-33.

11 Mooney J, Sellers SL, Blanke P, et al. CT-Defined Prosthesis-Patient Mismatch Downgrades Frequency and Severity, and Demonstrates No Association With Adverse Outcomes After Transcatheter Aortic Valve Replacement. JACC Cardiovasc Interv 2017;10:1578-87.

12 Pibarot P, Weissman NJ, Stewart WJ, et al. Incidence and sequelae of prosthesis-patient mismatch in transcatheter versus surgical valve replacement in high-risk patients with severe aortic stenosis: a PARTNER trial cohort--a analysis. J Am Coll Cardiol 2014;64:1323-34.

13 Stamou SC, Chen K, James TM, et al. Predictors and outcomes of patient-prosthesis mismatch after transcatheter aortic valve replacement. J Card Surg 2020;35:360-6.

14 Miyasaka M, Tada N, Taguri M, et al. Incidence, predictors, and clinical impact of prosthesis-patient mismatch following 
transcatheter aortic valve replacement in Asian patients: the OCEAN-TAVI registry. JACC Cardiovasc Interv 2018;11:771-80.

15 Kano S, Yamamoto M, Shimura T, et al. Gait speed can predict advanced clinical outcomes in patients who undergo transcatheter aortic valve replacement: insights from a Japanese multicenter registry. Circ Cardiovasc Interv 2017;10.

16 Kappetein AP, Head SJ, Généreux P, et al. Updated standardized endpoint definitions for transcatheter aortic valve implantation: the valve academic research Consortium-2 consensus document. J Am Coll Cardiol 2012;60:1438-54.

17 Singh M, Sethi A, Mishra AK, et al. Echocardiographic imaging challenges in obesity: guideline recommendations and limitations of adjusting to body size. J Am Heart Assoc 2020;9:e014609.

18 ROSENBAUM PR, Rubin DB. The central role of the propensity score in observational studies for causal effects. Biometrika 1983;70:41-55.

19 Alkhouli M, Holmes DR, Carroll JD, et al. Racial disparities in the utilization and outcomes of TAVR: TVT Registry report. JACC Cardiovasc Interv 2019;12:936-48.

20 Abdelghani M, Allali A, Kaur J, et al. Impact of prosthesis-iteration evolution and sizing practice on the incidence of prosthesis-patient mismatch after transcatheter aortic valve replacement. Catheter Cardiovasc Interv 2019;93:971-9.

21 Hahn RT, Pibarot P, Leipsic J, et al. The Effect of Post-Dilatation on Outcomes in the PARTNER 2 SAPIEN 3 Registry. JACC Cardiovasc Interv 2018;11:1710-8.

22 Yang T-H, Webb JG, Blanke P, et al. Incidence and severity of paravalvular aortic regurgitation with multidetector computed tomography nominal area oversizing or undersizing after transcatheter heart valve replacement with the Sapien 3: a comparison with the Sapien XT. JACC Cardiovasc Interv 2015;8:462-71.

23 Kazuno $\mathrm{Y}$, Maeno $\mathrm{Y}$, Kawamori $\mathrm{H}$, et al. Comparison of SAPIEN 3 and SAPIEN XT transcatheter heart valve stent-frame expansion: evaluation using multi-slice computed tomography. Eur Heart $J$ Cardiovasc Imaging 2016;17:1054-62.

24 Miyasaka M, Yoon S-H, Sharma RP, et al. Clinical outcomes of transcatheter aortic valve implantation in patients with extremely large annulus and SAPIEN 3 dimensions based on post-procedural computed tomography. Circ J 2019;83:672-80.

25 Saia F, Gandolfo C, Palmerini T, et al. In-hospital and thirty-day outcomes of the SAPIEN 3 ultra balloon-expandable transcatheter aortic valve: the S3U registry. Eurolntervention 2020;15:1240-7.

26 Watanabe Y, Hayashida K, Takayama M, et al. First direct comparison of clinical outcomes between European and Asian cohorts in transcatheter aortic valve implantation: the Massy Study Group vs. the PREVAIL JAPAN trial. J Cardiol 2015;65:112-6.

27 Yoon S-H, Ohno Y, Araki M, et al. Comparison of aortic root anatomy and calcification distribution between Asian and Caucasian patients who underwent transcatheter aortic valve implantation. Am J Cardiol 2015;116:1566-73.

28 Mack MJ, Leon MB, Thourani VH, et al. Transcatheter aortic-valve replacement with a balloon-expandable valve in low-risk patients. $N$ Engl J Med 2019;380:1695-705. 